\title{
Utility of a Cognitive-Behavioral Model to Predict Fatigue Following Breast Cancer Treatment
}

\author{
Kristine A. Donovan \\ H. Lee Moffitt Cancer Center \& Research Institute
}

\author{
Brent J. Small \\ H. Lee Moffitt Cancer Center \& Research Institute and \\ University of South Florida \\ Pamela Munster \\ H. Lee Moffitt Cancer Center \& Research Institute
}

Michael A. Andrykowski

University of Kentucky

\author{
Paul B. Jacobsen \\ H. Lee Moffitt Cancer Center \& Research Institute and University of South Florida
}

\begin{abstract}
The objective of the current study was twofold: (a) to determine whether subgroups of breast cancer patients could be identified on the basis of their distinct trajectory or pattern of fatigue following treatment for early stage cancer using growth mixture modeling and (b) to examine whether the subgroups could be distinguished on the basis of a cognitive-behavioral model. Growth mixture modeling and a prospective longitudinal design were used to examine the course of fatigue after treatment for early stage breast cancer. Women $(n=261$; mean age $=55.2$ years $)$ provided fatigue ratings for 6 months following treatment. A low-fatigue group $(n=85)$ and a high-fatigue group $(n=$ 176) were extracted. Women who were not married, had a lower income, had a higher body mass index, engaged in greater fatigue catastrophizing, and were lower in exercise participation were more likely to be in the high-fatigue group. Only body mass index and catastrophizing remained significant predictors in multivariate analysis. Findings suggest considerable heterogeneity in the experience of fatigue following treatment and support the utility of a cognitive-behavioral model in predicting the course of posttreatment fatigue.
\end{abstract}

Keywords: fatigue, breast cancer, catastrophizing, exercise, growth mixture modeling

Fatigue is one of the most common symptoms reported by women undergoing chemotherapy or radiotherapy for breast cancer and may persist for months and even years following treatment (Andrykowski, Curran, \& Lightner, 1998; Bower et al., 2006; Broeckel, Jacobsen, Horton, Balducci, \& Lyman, 1998; Jacobsen \& Stein, 1999; Servaes, S. Verhagen, \& Bleijenberg, 2002). Evidence for the persistence of fatigue comes primarily from studies comparing breast cancer patients with age-matched women with

Kristine A. Donovan, Health Outcomes and Behavior Program, H. Lee Moffitt Cancer Center \& Research Institute, Tampa, Florida; Brent J. Small, Health Outcomes and Behavior Program, H. Lee Moffitt Cancer Center \& Research Institute, and School of Aging Studies, University of South Florida; Michael A. Andrykowski, Department of Behavioral Science, College of Medicine, University of Kentucky; Pamela Munster, Breast Cancer Program, H. Lee Moffitt Cancer Center \& Research Institute; Paul B. Jacobsen, Health Outcomes and Behavior Program, H. Lee Moffitt Cancer Center \& Research Institute, and Department of Psychology, University of South Florida.

This research was supported by National Cancer Institute Grant R01 CA82822. We thank Christine Marsella for her editorial assistance.

Correspondence concerning this article should be addressed to Kristine A. Donovan, Health Outcomes and Behavior Program, H. Lee Moffitt Cancer Center \& Research Institute, 12902 Magnolia Drive, MRC-PSY, Tampa, Florida 33612. E-mail: donovaka@moffitt.usf.edu no history of cancer (Andrykowski et al., 1998; Broeckel et al., 1998; Hann et al., 1998) and with published norms (Bower et al., 2000). On the basis of the research to date, however, it is difficult to draw definitive conclusions about fatigue persistence. This is because of the cross-sectional nature of most previous studies and because the length of time between treatment completion and the assessment of fatigue has varied greatly across previous studies. An additional issue concerns the fact that most studies consider only fatigue scores in the aggregate and do not examine whether there are subgroups of patients who differ in their experience of fatigue after treatment completion. This approach of using aggregate (i.e., group mean) scores may mask meaningful differences among subgroups of patients in the severity, course, and predictors of fatigue. For example, it may be the case that some patients report a reduction in fatigue as time since treatment increases, whereas others report worsening of fatigue; consequently, the overall trajectory of fatigue for the group as a whole would not appear to change.

A study of fatigue in breast cancer patients by Bower et al. (2000) partly addressed this issue. In this study, women diagnosed with breast cancer an average of 35 months previously who scored at or below the midpoint of the Medical Outcomes Study energy/ fatigue subscale were classified as fatigued and compared with women who scored above the midpoint and were classified as 
nonfatigued. Women classified as fatigued were more likely to be younger, to have a lower annual income, to not be married or in a relationship, and to have higher rates of arthritis, headache, and heart disease. With respect to treatment, women in the fatigued group were more likely to have been treated with chemotherapy. They also reported significantly more pain, menopausal symptoms, problems with sleep, and depressive symptoms than did the nonfatigued women. In a similar effort, Servaes, C. A. Verhagen, and Bleijenberg (2002) compared fatigue in breast cancer patients who had completed treatment between 6 and 70 months previously and women with a diagnosis of chronic fatigue syndrome. The researchers identified a subset of breast cancer patients who reported a level of fatigue and impairment comparable to that seen in women with chronic fatigue syndrome. Compared with the other breast cancer survivors, this subset of women scored higher on measures of depression, interpersonal sensitivity, and obsessivecompulsive behavior.

These results suggest that woman may vary considerably in their experience of fatigue following breast cancer treatment. However, it is difficult to draw definitive conclusions from the existing research because of the cross-sectional nature of the studies and the inconsistent definitions of significant fatigue. We sought to address these shortcomings by assessing fatigue longitudinally in women during the 6-month period following completion of treatment for early stage breast cancer. The first 6 months following treatment were examined because it is during this time period that many women attempt to resume their usual activities and expect some resolution of their fatigue. To determine whether subgroups of women who differ with respect to their fatigue scores following treatment could be identified, we examined changes in fatigue scores over time using growth mixture modeling. We also evaluated the utility of a cognitive-behavioral model in predicting the experience of fatigue following treatment.

\section{Growth Mixture Modeling}

In the current study, we used the growth mixture modeling technique to examine longitudinal changes in fatigue following the end of treatment for breast cancer and to determine whether subgroups of women exist that differ in their levels of fatigue following treatment. This approach is capable of detecting not only whether a group of persons changes on some outcome measure but whether there are individual differences in the rates of change among group members (e.g., Nesselroade \& Baltes, 1979). The widespread application of random effects and mixed model approaches to data analysis reflects this interest and has made addressing these types of questions more accessible (see Singer \& Willett, 2003, for a review). The main advantage of these techniques is the estimation of individually varying trajectories of change for the outcome measure. Thus, these approaches embrace the fact that people begin and end in different places in terms of their scores on a measure over the longitudinal assessment period.

The mixed model approaches to data analysis described above acknowledge the heterogeneity in change over time but assume that the individuals who are varying are also derived from the same population. By contrast, growth mixture modeling does not require this assumption be held and, in fact, allows for the identification of subpopulations or groups of individuals through the application of a latent class membership variable (for reviews, see Jones, Nagin,
\& Roeder, 2001; Muthén, 2004). To some extent, this approach evaluates whether there is sufficient homogeneity in the heterogeneity of change over time to allow the identification of classes of individuals who differ in terms of their initial starting points and/or rates of change over time.

\section{Cognitive-Behavioral Model}

Wessely and colleagues (Wessely, Hotopf, \& Sharpe, 1998) have proposed a conceptual model that can be used to explain the persistence of fatigue in individuals with chronic medical illness The essential feature of this model is the distinction drawn between factors that precipitate fatigue initially and those that perpetuate fatigue later in the course of the illness. Factors that may precipitate fatigue include acute manifestations of illness (e.g., infection and fever) and acute treatment side effects (e.g., pain and nausea). Factors that may perpetuate fatigue include specific cognitive and behavioral responses to fatigue and the physiological changes that may accompany these responses. In keeping with this conceptual model, we developed a cognitive-behavioral model that identifies catastrophizing as a cognitive factor and lack of physical activity as a behavioral factor that may perpetuate fatigue following treatment for breast cancer. Several lines of evidence provide preliminary support for this model. First, there is evidence that breast cancer patients who engage in more catastrophizing about fatigue experience greater fatigue and poorer quality of life (Broeckel et al., 1998; Jacobsen, Andrykowski, \& Thors, 2004; Jacobsen, Azzarello, \& Hann, 1999). Second, there is evidence that among breast cancer patients, greater physical activity during treatment is related to lower levels of fatigue (Berger, 1998; Berger \& Higginbotham, 2000; Mock et al., 2001). Finally, there is preliminary evidence that exercise interventions are effective in relieving fatigue in breast cancer survivors (Courneya et al., 2003).

\section{Current Study}

The current study had two specific aims. The first was to determine whether we could identify subgroups of patients on the basis of their distinct trajectory or pattern of fatigue following treatment using growth mixture modeling. We hypothesized that at least two subgroups of women could be identified who differed in their experience of fatigue following treatment completion. The second aim was to examine whether the subgroups could be distinguished on the basis of a cognitive-behavioral model. Specifically, we hypothesized that differences in catastrophizing and exercise activity measured at treatment completion would predict different trajectories or patterns of fatigue in the subsequent 6 months.

\section{Method}

\section{Participants}

Participants were women with early stage breast cancer scheduled to be treated with chemotherapy followed by radiotherapy (CT + RT group) or radiotherapy only (RT group) at the H. Lee Moffitt Cancer Center at the University of South Florida or at the Comprehensive Breast Care Center of the Lucille Parker Markey Cancer Center at the University of Kentucky. Eligibility criteria were that participants (a) be at least 18 years of age; (b) have no 
documented or observable psychiatric or neurological disorders that would interfere with study participation (e.g., dementia or psychosis); (c) be able to speak and read standard English; (d) have no history of cancer other than basal cell skin carcinoma; (e) be diagnosed with stage 0 , I, or II breast cancer; (f) have been treated surgically with lumpectomy or mastectomy; (g) be scheduled to receive a minimum of four cycles of chemotherapy and then radiotherapy following surgery (CT $+\mathrm{RT}$ group) or be scheduled to receive only radiotherapy following surgery (RT group); (h) have no prior history of treatment with chemotherapy or radiotherapy; (i) have no other chronic or life-threatening diseases in which fatigue is a prominent symptom (e.g., AIDS, multiple sclerosis, or chronic fatigue syndrome); and (j) provide written informed consent.

\section{Procedure}

Eligibility was determined by chart review and consultation with the attending physician. Eligible patients were recruited and informed consent was obtained during an outpatient clinic visit prior to the start of chemotherapy (CT + RT group) or radiotherapy (RT group). Those women who provided informed consent completed a questionnaire assessing demographic characteristics prior to beginning treatment. Fatigue and psychosocial factors were assessed at the end of treatment, and 2, 4, and 6 months after treatment.

A total of 309 women completed the assessment at the end of treatment. Of these, 48 women voluntarily withdrew during the 6 -month posttreatment period. The remaining 261 women were the focus of the current study. Comparisons between women included in the analyses and those not included revealed no statistically significant differences $(p<.05)$ in terms of demographic or clinical characteristics. Of the 261 women included in the analyses, 221 had complete fatigue data across the four measurement points, 25 women were missing one assessment, 13 were missing two assessments, and 2 contributed only one assessment to the analyses.

\section{Measures}

Demographic data. Demographic data obtained pretreatment via self-report included age, race or ethnicity, marital status, annual household income, and educational level.

Clinical data. Variables assessed via chart review before treatment included disease stage, type of breast surgery, height, and weight. Comorbid medical conditions were also assessed at this time with a self-report version of the Charlson Comorbidity Index (Katz, Chang, Sangha, Fossel, \& Bates, 1996). Types of chemotherapy agents (CT + RT group), cumulative radiation doses (CT + RT and RT groups), and hormone therapy status (CT + RT and RT groups) were recorded following treatment.

Fatigue. The Fatigue Symptom Inventory (Hann et al., 1998), a 14-item measure that assesses the frequency and severity of fatigue and its perceived disruptiveness, was administered at all four time points. Analyses focused on the average of four items assessing fatigue severity. Participants rated on 11-point scales $(0=$ not at all fatigued, $10=$ as fatigued as I could be $)$ their level of fatigue (a) on the day they felt most fatigued in the past week, (b) on the day they felt least fatigued in the past week, (c) on average in the past week, and (d) right now. This average fatigue score showed high internal consistency across the four measurement points ( $\alpha=.91-.93$ ). Previous research has demonstrated the reliability and validity of the Fatigue Symptom Inventory in women diagnosed with breast cancer (Broeckel et al., 1998; Hann et al., 1998).

Catastrophizing. The Catastrophizing scale of the Cognitive Coping Strategies Inventory (Butler, Damarin, Beaulieu, Schwebel, \& Thorn, 1989) was designed to measure catastrophizing in response to pain. The Fatigue Catastrophizing Scale was developed by substituting the word fatigue for pain in the instructions and item content (Jacobsen et al., 1999). Respondents indicated on a 5 -point scale $(1=$ never true, $5=$ all of the time true $)$ how often each item was true when they were fatigued. The 10 items composing this scale reflect tendencies to engage in negative self-statements and overly negative thoughts about the future (e.g., "I find myself expecting the worst when I'm fatigued"). The Fatigue Catastrophizing Scale has demonstrated high internal consistency $(\alpha=.92)$ in previous research with women diagnosed with breast cancer (Jacobsen et al., 1999). Validity has been demonstrated by research showing that among breast cancer patients, greater catastrophizing about fatigue is associated with greater fatigue and poorer quality of life (Broeckel et al., 1998; Jacobsen et al., 1999). This measure was administered at the end of treatment.

Exercise activity. The Leisure Score Index of the Godin Leisure-Time Exercise Questionnaire (Godin, Jobin, \& Bouillon, 1986) assesses the average frequency of mild, moderate, and strenuous exercise in the past week. These frequencies are multiplied by 3,5 , and 9, respectively, and total weekly activity is calculated by summing the products (Godin, Jobin, \& Bouillon, 1986). The reliability and validity of the Leisure Score Index has been found to compare favorably with other self-report measures of exercise in terms of test-retest scores and correlations with objective activity monitors and objective fitness indexes (Jacobs, Ainsworth, Hartman, \& Leon, 1993). This measure was administered at the end of treatment.

\section{Statistical Analysis}

A multistage procedure was used in which the growth mixture models were applied to the longitudinal fatigue data and then demographic, medical, and psychosocial measures assessed at the end of treatment were evaluated as potential predictors of differences in fatigue group membership. Growth mixture model analysis is an iterative procedure in which the analyses began with a one-class model, and then successive models extracted additional classes. The one-class model is analogous to a standard latent growth or random effects model in which all participants are assumed to be derived from the same population. With information from statistical tests for each parameter, a determination can be made concerning whether changes over the follow-up interval are best construed as linear or quadratic in nature. Following analysis of the one-class model, a two-class model was examined, followed by a three-class model, and so on. At each successive model, several statistical fit parameters were inspected (i.e., log likelihood [-2LL], Akaike information criterion [AIC], and Bayesian information criterion [BIC]), with lower values indicating a better fitting model. At the point at which the statistical fit parameters 
stopped improving or indicated a loss of statistical fit, the iterative model testing procedure was suspended. In addition to the statistical index of model fit, the theoretical relevance of the additional classes of participants was evaluated. Recent evidence (e.g., Bauer \& Curran, 2003; but for commentary, see Cudeck \& Henly, 2003; Muthén, 2003; Rindskopf, 2003) suggests that on the basis of statistical considerations alone, growth mixture modeling procedures may derive too many classes of participants. Mplus (Version 3.12; Muthén \& Muthén, 2004) was used to develop the growth curve models and the growth mixture models.

Following the growth mixture analysis, we examined differences in class membership as a function of demographic, clinical, and psychosocial factors. The demographic measures included age, years of education, race or ethnicity, income, and marital status. The relevant clinical characteristics were treatment type (CT + RT, RT), disease stage (stage 0-I, stage II), lumpectomy surgery (yes, no), hormone therapy (yes, no), body mass index, and comorbidity index scores. The psychosocial factors examined were fatigue catastrophizing and physical exercise at the end of treatment. To examine the potential of these variables to predict fatigue class membership, we computed univariate and multivariate logistic regression analyses with fatigue class membership as the outcome measure. For the multivariate logistic regression, only measures that were statistically significant in the univariate analyses were used as predictors of class membership.

\section{Results}

\section{Growth Models of Change}

Across the 6-month follow-up period, average fatigue scores declined by almost one full point (end of treatment, $M=3.19$, $S D=2.37 ; 2$ months posttreatment, $M=2.33, S D=2.14 ; 4$ months posttreatment, $M=2.24, S D=2.11 ; 6$ months posttreatment, $M=2.25, S D=2.24)$. Means and standard deviations for the individual scores used to derive the average fatigue scores appear in Table 1. An initial one-class growth mixture model specifying a linear change slope was estimated, with time indexed by a centered variable for months following the end of treatment.
The results of the linear slope model $(-2 \mathrm{LL}(d f=6)=1,976.61$; $\mathrm{AIC}=3,965.22 ; \mathrm{BIC}=3,986.61)$ indicated a significant intercept (estimate $=2.52, S E=0.11, p<.001$ ), as well as a negative slope of -.15 (estimate $=-.15, S E=.02, p<.001$ ). These results show that fatigue scores declined by an average of 0.15 points per month following treatment. The next model tested included both a linear and a quadratic slope component. This model provided a better fit to the data $(-2 \mathrm{LL}(d f=10)=1,959.79, \Delta-2 \mathrm{LL}(\Delta d f=$ $4)=16.82 ; \mathrm{AIC}=3,939.58, \Delta \mathrm{AIC}=25.64 ; \mathrm{BIC}=3,975.22$, $\Delta \mathrm{BIC}=11.39)$. The results indicated a statistically significant intercept (estimate $=2.26, S E=0.13, p<.001$ ), as well as statistically significant negative linear slope (estimate $=-.14$, $S E=.02, p<.001)$ and a positive quadratic slope (estimate $=.05$, $S E=.01, p<.01$ ). The results of this analysis (see Figure 1 , Overall-Predicted model line) show that participants' average fatigue rating at the end of treatment was approximately 3 and declined by almost 1 point across the following 4 months, when improvement in fatigue scores reached a plateau.

The growth mixture analyses began with the linear and quadratic model described above and tested a model in which two classes could be extracted from the data. The results from the fit statistics indicated that this model provided a better fit to the data, as compared with the one-class linear and quadratic model (-2LL $(d f=20)=1,804.64, \Delta-2 \mathrm{LL}(\Delta d f=10)=155.15 ; \mathrm{AIC}=$ $3,649.29, \Delta \mathrm{AIC}=290.29 ; \mathrm{BIC}=3,720.58, \Delta \mathrm{BIC}=254.64)$. The first class (low-fatigue group) comprised 85 individuals with an intercept of approximately 0.5 (estimate $=0.53, S E=0.09, p<$ .001 ), a statistically significant negative linear slope of .06 points per month (estimate $=-.06, S E=.01, p<.001$ ), but a quadratic slope that was not statistically significant (estimate $=.004, S E=$ $.007, p>.05$ ). The second class (high-fatigue group) consisted of 176 persons whose intercept was approximately 3 points (estimate $=3.08, S E=0.16, p<.001)$, a negative linear slope of almost -.2 units per month (estimate $=-.18, S E=.03, p<.001$ ) and a statistically significant positive quadratic slope of .07 (estimate $=.07, S E=.02, p<.01)$. A three-class mixture model was then fit to the data; however, this model did not provide any increase in the statistical fit of the model $(-2 \mathrm{LL}(d f=30)=$

Table 1

Mean ( $\pm S D)$ for Individual Fatigue Symptom Index Scores Across Time and Fatigue Class Membership

\begin{tabular}{lcccc}
\hline Fatigue class & Time 1 & Time 2 & Time 3 & Time 4 \\
\hline $\begin{array}{l}\text { Most fatigue } \\
\text { Low class }\end{array}$ & $1.44 \pm 1.19$ & $1.11 \pm 1.24$ & $1.10 \pm 1.31$ & $0.78 \pm 0.91$ \\
$\quad$ High class & $6.07 \pm 2.39$ & $4.71 \pm 2.85$ & $4.52 \pm 2.81$ & $4.52 \pm 2.72$ \\
Least fatigue & & & \\
$\quad$ Low class & $0.32 \pm 0.59$ & $0.17 \pm 0.41$ & $0.17 \pm 0.41$ & $0.11 \pm 0.32$ \\
$\quad$ High class & $2.84 \pm 2.05$ & $2.06 \pm 2.13$ & $1.90 \pm 1.92$ & $2.17 \pm 2.25$ \\
Average fatigue & $0.79 \pm 0.86$ & $0.59 \pm 0.72$ & $0.52 \pm 0.69$ & $0.43 \pm 0.52$ \\
$\quad$ Low class & $4.34 \pm 2.08$ & $3.16 \pm 2.28$ & $3.12 \pm 2.16$ & $3.23 \pm 2.34$ \\
$\quad$ High class & & $0.33 \pm 0.63$ & $0.38 \pm 0.62$ & $0.15 \pm 0.39$ \\
Current fatigue & $0.43 \pm 0.67$ & $2.95 \pm 2.56$ & $2.86 \pm 2.61$ & $2.80 \pm 2.46$ \\
$\quad$ Low class & $4.02 \pm 2.61$ & & & \\
$\quad$ High class & & &
\end{tabular}

Note. Fatigue was rated on a scale of 0 (not at all fatigued) to 10 (as fatigued as I could be). Time $1=$ end of treatment; Time $2=2$ months posttreatment; Time $3=4$ months posttreatment; Time $4=6$ months posttreatment. 


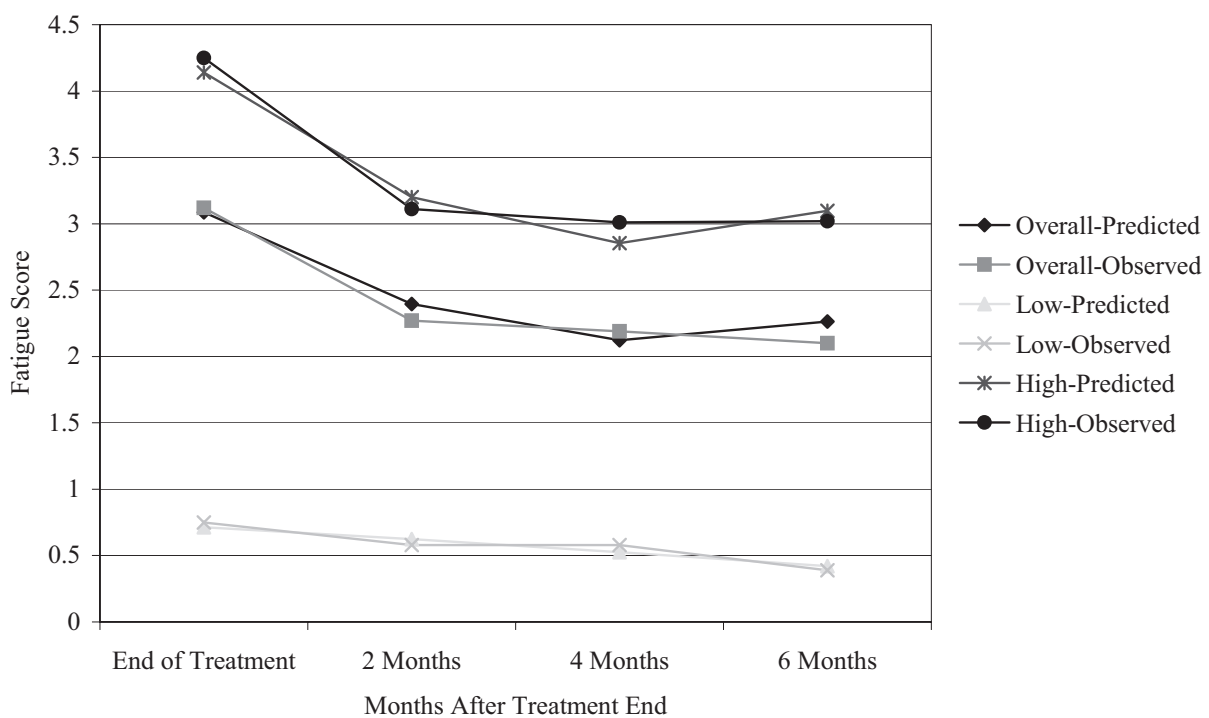

Figure 1. One-class and two-class growth mixture models of fatigue across the 6-month period following the end of treatment.

1,798.47, $\Delta-2 \mathrm{LL}(\Delta d f=10)=6.17 ; \mathrm{AIC}=3,656.94, \Delta \mathrm{AIC}=$ $7.65 ; \mathrm{BIC}=3,673.88, \Delta \mathrm{BIC}=43.30)$. As a result, the linear and quadratic two-class model described above was accepted as final. ${ }^{1}$

The longitudinal trajectories for the two-class model are shown in Figure 1. For both sets of classes and the single class (overall) model, two sets of lines are presented. The predicted scores are values derived from the statistical parameter estimates, whereas the observed scores represent the raw mean values. The figure indicates that the low-fatigue group consisted of persons who had relatively low ratings of fatigue at the end of treatment, less than 1 point, and their ratings exhibited a slow linear decline over time. By contrast, the high-fatigue group exhibited much higher ratings at end of treatment, over 4 points, and exhibited a decline across the follow-up period, although the magnitude of improvement lessened between 4 and 6 months following treatment. As seen in the figure, there is a high degree of concordance between the observed raw scores and those predicted by the statistical models. Another metric for evaluating the adequacy of the model is the $U$ statistic (Kaplan \& George, 1998; Theil, 1966). In the current analysis, the $U$ statistic, with values approaching 0 indicating a high degree of concordance between the observed and predicted scores, indicated that the growth trajectories for the low-fatigue class $(U=.038)$ and the high-fatigue class $(U=.025)$ provide a good match to the observed data.

\section{Predictors of Class Membership}

Table 2 shows the demographic, clinical, and psychosocial characteristics for the entire sample and for the two classes of participants identified in the growth mixture analyses. Univariate logistic regression analyses revealed that several of the predictor variables were significantly associated with fatigue class membership. Specifically, unmarried status, lower income, higher body mass index, higher scores on fatigue catastrophizing, and lower physical activity ratings were significantly $(p \leq .05)$ associated with membership in the high-fatigue class.
Finally, all variables that were statistically significant $(p<.05)$ in the univariate logistic regressions were entered into a multivariate logistic regression. The results shown in Table 3 indicate that only two measures remained as significant predictors, body mass index and fatigue catastrophizing. Consistent with the univariate analyses, having a higher body mass index and having higher fatigue catastrophizing scores at the end of treatment were associated with membership in the higher fatigue group.

\section{Discussion}

The first aim of the current study was to determine whether we could identify subgroups of patients on the basis of their pattern of fatigue following treatment for breast cancer. The results indicated that two groups of patients could be identified. One group reported relatively low levels of fatigue immediately after completing treatment. Over the next 6 months, these low levels declined slightly. By contrast, the other group reported much higher levels of fatigue immediately after treatment. Although they too demonstrated a decline in fatigue during the follow-up period, this improvement

\footnotetext{
${ }^{1}$ In addition to the final model reported here, analyses also were conducted in which the fatigue scores obtained just before the start of chemotherapy were used as a covariate, to examine whether differences at the start of treatment influenced fatigue group membership. These analyses indicated that pretreatment fatigue scores were related to the posttreatment fatigue intercept value but not to the rate of linear or quadratic change. As such, the covariate simply acted as a constant that was subtracted from fatigue scores at a later point. Inclusion of the covariate also did not influence fatigue group classification; there was $96.4 \%$ overlap between patients who were classified as low-fatigue group with or without the covariate and $99.4 \%$ overlap between patients who were classified as high-fatigue group with or without the covariate. For ease of interpretation, results without the use of the covariate are presented in Figure 1. The values represent ratings from patients across the period following the end of treatment rather than these values minus a baseline covariate.
} 
Table 2

Sample Description and Univariate Predictors of Class Membership

\begin{tabular}{|c|c|c|c|c|c|c|}
\hline \multirow[b]{2}{*}{ Characteristic } & \multirow[b]{2}{*}{$\begin{array}{c}\text { Total } \\
(N=261)\end{array}$} & \multicolumn{2}{|c|}{ Fatigue class } & \multicolumn{3}{|c|}{ Univariate logistic regression } \\
\hline & & $\begin{array}{c}\text { Low } \\
(n=85)\end{array}$ & $\begin{array}{c}\text { High } \\
(n=176)\end{array}$ & OR & $95 \% \mathrm{CI}$ & $p$ \\
\hline \multicolumn{7}{|c|}{ Demographic characteristics } \\
\hline \multicolumn{7}{|l|}{ Age (years) } \\
\hline$M$ & 55.16 & 54.48 & 55.48 & 1.01 & $0.98,1.00$ & .439 \\
\hline$S D$ & 9.79 & 14.44 & 9.70 & & & \\
\hline \multicolumn{7}{|l|}{ Race or ethnicity } \\
\hline \% Caucasian & 91.5 & 95.2 & 89.8 & 2.27 & $0.75,6.96$ & .148 \\
\hline$\%$ other & 8.5 & 4.8 & 10.2 & & & \\
\hline \multicolumn{7}{|l|}{ Education (years) } \\
\hline$M$ & 14.62 & 14.44 & 14.71 & 1.04 & $0.93,1.16$ & .500 \\
\hline$S D$ & 2.63 & 2.32 & 2.77 & & & \\
\hline \multicolumn{7}{|l|}{ Marital status } \\
\hline$\%$ married & 72.3 & 82.1 & 67.6 & 2.20 & $1.16,4.19$ & .016 \\
\hline \multicolumn{7}{|l|}{ Annual household income } \\
\hline$\%>\$ 40,000 /$ year & 68.5 & 77.3 & 64.4 & 0.53 & $0.28,0.99$ & .048 \\
\hline \multicolumn{7}{|c|}{ Clinical characteristics } \\
\hline \multicolumn{7}{|l|}{ Treatment group } \\
\hline$\% \mathrm{CT}+\mathrm{RT}$ & 43.3 & 47.1 & 41.5 & 0.79 & $0.47,1.34$ & .394 \\
\hline \multicolumn{7}{|l|}{ Disease stage } \\
\hline$\%$ Stage 0 or 1 & 63.9 & 60.2 & 65.7 & 1.26 & $0.74,2.17$ & .396 \\
\hline \multicolumn{7}{|l|}{ Surgery type } \\
\hline$\%$ lumpectomy only & 95.6 & 96.3 & 95.3 & 0.78 & $0.20,3.02$ & .718 \\
\hline \multicolumn{7}{|l|}{ Current hormone therapy } \\
\hline$\%$ yes & 62.8 & 59.8 & 65.5 & 0.78 & $0.45,1.35$ & .381 \\
\hline \multicolumn{7}{|l|}{ Body mass index } \\
\hline$M$ & 27.11 & 25.67 & 27.79 & 1.07 & $1.02,1.13$ & .009 \\
\hline$S D$ & 6.00 & 5.53 & 6.11 & & & \\
\hline \multicolumn{7}{|c|}{ Charlson Comorbidity Index } \\
\hline$M$ & 2.37 & 2.27 & 2.42 & 1.30 & $0.87,1.95$ & .198 \\
\hline$S D$ & 0.79 & 0.68 & 0.83 & & & \\
\hline
\end{tabular}

Psychosocial characteristics

\begin{tabular}{|c|c|c|c|c|c|c|}
\hline \multicolumn{7}{|c|}{ Fatigue catastrophizing } \\
\hline$M$ & 1.27 & 1.09 & 1.36 & 39.69 & $8.29,189.89$ & $<.001$ \\
\hline$S D$ & 0.40 & 0.17 & 0.45 & & & \\
\hline \multicolumn{7}{|c|}{ Exercise activity } \\
\hline$M$ & 16.01 & 20.07 & 14.18 & 0.99 & $0.97,1.00$ & .045 \\
\hline$S D$ & 20.44 & 24.32 & 18.22 & & & \\
\hline
\end{tabular}

Note. $\mathrm{OR}=$ odds ratio; $\mathrm{CI}=$ confidence interval; $\mathrm{CT}+\mathrm{RT}=$ treated with chemotherapy followed by radiotherapy.

did not persist between 4 and 6 months, and at 6 months this group continued to report relatively high levels of fatigue.

The identification of two groups of patients with distinct patterns of fatigue is consistent with the findings of Helgeson, Snyder, and Seltman (2004). In this study, the authors assessed women's psychological and physical adjustment to breast cancer over 4 years. Although the majority of the women showed fairly consistent improvement in mental and physical functioning over time, the researchers identified subgroups of women who showed distinct patterns of change. For example, with respect to changes in physical functioning, one group demonstrated better physical functioning at baseline (4 months postdiagnosis), improved slightly over time, and remained high until the end of the follow-up period. Another group demonstrated a lower level of physical functioning at baseline and did not change over time. Helgeson et al. (2004) also found that personal and social resources, but not demographic or disease variables, distinguished the different trajectories or patterns of mental and physical functioning.

The second aim of the study was to examine whether the subgroups identified could be distinguished on the basis of demographic, clinical, and psychosocial factors. In univariate analysis, the extent of catastrophizing at treatment completion predicted different trajectories of fatigue (i.e., group membership) across the 6-month follow-up period. As hypothesized, higher fatigue catastrophizing scores at the end of treatment predicted membership in the high-fatigue group. Physical exercise was a statistically significant predictor ( $p=.045$ ) of fatigue group membership in the univariate analysis, providing support for the hypothesis that less 
Table 3

Multivariate Logistic Regression Predicting Fatigue Class Membership

\begin{tabular}{lrlr}
\hline \multicolumn{1}{c}{ Variable } & OR & \multicolumn{1}{c}{$95 \% \mathrm{CI}$} & \multicolumn{1}{c}{$p$} \\
\hline Marital status & 1.79 & $0.76,4.05$ & .158 \\
Income & 1.31 & $0.60,2.85$ & .504 \\
Body mass index & 1.08 & $1.01,1.15$ & .023 \\
Fatigue catastrophizing & 20.76 & $4.19,102.89$ & $<.001$ \\
Exercise activity & 0.99 & $0.98,1.01$ & .202 \\
\hline
\end{tabular}

Note. $\mathrm{OR}=$ odds ratio $\mathrm{CI}=$ confidence interval.

physical activity at the end of treatment would be associated with more fatigue in the posttreatment period. Higher body mass index at treatment completion also emerged as a significant univariate predictor of membership in the high-fatigue group. Marital status and annual income also distinguished different trajectories of fatigue in univariate analysis. Women who were not married and had lower incomes were more likely to be in the high-fatigue group. In the multivariate analysis, only fatigue catastrophizing and body mass index remained as statistically significant predictors of fatigue group membership across the 6-month follow-up period.

In addition to identifying groups of women who differ significantly in their experience of fatigue following treatment for breast cancer, findings regarding the role of certain factors in perpetuating fatigue in the posttreatment period partially validate the proposed cognitive-behavioral model. Previous research has suggested that catastrophizing about fatigue may be a risk factor for greater fatigue after treatment (Broeckel et al., 1998; Jacobsen et al., 2004; Jacobsen et al., 1999). However, with the exception of one study (Jacobsen et al., 2004), the existing research has been cross-sectional in design. The results of the current study indicate that catastrophizing about fatigue during the active treatment period has implications for the experience of fatigue following treatment completion. There is a growing body of research documenting that physical activity has a positive effect on fatigue in individuals with cancer. Researchers have found that individuals who report less physical activity during or after treatment report greater levels of fatigue (Berger, 1998; Berger \& Higginbotham, 2000; Dimeo et al., 1997; Schwartz, 1998). Likewise, there is growing evidence that interventions designed to increase activity levels have had beneficial effects on fatigue in cancer patients (for a review, see Irwin \& Ainsworth, 2004). In this study, however, increased physical activity at the time of treatment completion was a significant predictor of lower levels of fatigue in the subsequent 6-month period in the univariate analysis but did not remain a significant predictor in the multivariate analysis.

As noted previously, several other factors besides catastrophizing were significant predictors of fatigue group membership in univariate analysis. The relationship of marital status and income with fatigue observed in the present study is consistent with the findings of Bower et al. (2000) and de Jong, Candel, Schouten, Huijer Abu-Saad, and Courtens (2004). In general, however, previous research has not found a relationship between fatigue and demographic variables (for a review, see Servaes, C. Verhagen, \& Bleijenberg, 2002). In the current study, it may be the case that women who are married and have higher incomes have greater access to interpersonal and material resources that may assist them in ultimately reducing their fatigue. Previous studies investigating differences in fatigue among breast cancer survivors on the basis of treatment modality have yielded mixed results (Andrykowski et al., 1998; Berglund, Bolund, Fornander, Rutqvist, \& Sjoden, 1991; Bower et al., 2000; Woo, Dibble, \& Piper, 1998). In the current study, women who received both chemotherapy and radiotherapy were indistinguishable in terms of their fatigue class membership from women who received radiotherapy only. It may be the case that differences emerge only later than 6 months posttreatment. Consistent with previous research (e.g., Broeckel et al., 1998; Mast, 1998; Okuyama et al., 2000), we did not find a relationship between the use of hormone therapy and fatigue group membership. Although weight gain is a common problem among women treated with chemotherapy for early stage breast cancer (Kumar et al., 2004; Lankester, Phillips, \& Lawton, 2002; McInnes \& Knobf, 2001), we did not hypothesize that body mass index would be associated with fatigue following treatment. The few studies that have investigated this relationship have yielded mixed results (Andrykowski et al., 1998; Kumar et al., 2004; Wratten et al., 2004). The results of the current study, particularly the finding that body mass index was the only demographic and clinical variable to remain a significant predictor in the multivariate analysis, lend further support for the view that greater body mass index is significantly associated with worse fatigue following treatment.

The current study has important clinical implications. According to the National Comprehensive Cancer Network Clinical Practice Guidelines for Cancer-Related Fatigue (National Comprehensive Cancer Network, 2003), persons who rate their fatigue as 4 or higher on a 10-point scale deserve further evaluation, in terms of a focused history and physical examination. At 6 months following treatment, $44 \%$ of women in the high-fatigue group provided scores of 4 or greater on the item that assessed average fatigue in the past week, and $59 \%$ of women in the high-fatigue group provided scores of 4 or greater on the item that assessed most fatigue in the past week. By contrast, no members of the lowfatigue group rated their fatigue as 4 or higher on either item. Thus, the results indicate that not only are these groups statistically distinct, but a sizable proportion of the high-fatigue group merits further evaluation for their fatigue. To better appreciate the relative differences in fatigue, we also compared the fatigue reported by the high-fatigue group with self-reported fatigue scores from a sample of women $(n=85$; mean age $=57.4$ years $)$ with no history of cancer matched for age and geographic residence with participants from the current study. On the composite measure of the four fatigue items, this healthy noncancer group averaged $2.42(S D=$ $1.62)$, compared with $3.19(S D=2.19)$ for the high-fatigue group at the 6-month posttreatment follow-up. Similarly, whereas the healthy noncancer group reported an average fatigue score in the past week of $2.53(S D=1.94)$, the high-fatigue group reported an average level of fatigue of $3.23(S D=2.84)$.

A considerable body of research has documented a relationship between catastrophizing and the experience of pain (Sullivan \& D'Eon, 1990; Sullivan et al., 2001) and demonstrated that interventions based on cognitive-behavioral models can lead to reductions in catastrophizing that are associated with better adjustment to chronic pain (Jensen, Turner, \& Romano, 2001). Research in chronic pain also suggests that certain behaviors such as activity reduction are related to catastrophizing and may serve to exacer- 
bate pain. Taken together, these findings suggest that similar interventions designed to reduce reliance on catastrophizing and reduce weight may be effective in reducing fatigue in women completing treatment for early stage breast cancer. Accordingly, promoting increases in physical activity as a means of facilitating weight loss should be viewed as a potential intervention strategy. The current study also demonstrates the usefulness of growth mixture modeling for the longitudinal study of quality of life outcomes in cancer patients. As noted previously, similar methods have been used successfully to identify groups of breast cancer survivors who differ in their psychological and physical adjustment over time and to identify predictors of group membership (Helgeson et al., 2004). The pattern of results seen in the present study highlights that there are considerable individual differences in the way people experience fatigue following treatment for breast cancer. Statistical analytic techniques that permit examination and prediction of patterns of response over time, such as growth mixture modeling, are likely to prove useful for examining changes in other aspects of quality of life, such as sexual functioning and cognitive functioning.

Certain limitations of the current study should be noted. The women in our sample predominately were Caucasian, were married, and had annual household incomes over $\$ 40,000$. Whether our findings are generalizable to a more diverse population of women with early stage breast cancer survivors is not known. A strength of the current study is its longitudinal design and the repeated assessments during the posttreatment period. However, we assessed fatigue only until 6 months after treatment. Whether different patterns of fatigue emerge later in the course of cancer survivorship is unclear.

In conclusion, the present study identified two groups of breast cancer patients on the basis of their patterns of fatigue. One group experienced relatively low levels of fatigue at the end of treatment and throughout the follow-up period, whereas the other group experienced relatively high levels of fatigue at the end of treatment that persisted throughout the 6-month follow-up period. Consistent with a cognitive-behavioral model, patients in the high-fatigue group were more likely to rely on fatigue catastrophizing. These findings suggest that efforts should be aimed at the development and evaluation of interventions to reduce patients' reliance on catastrophizing as a cognitive coping strategy. Similarly, interventions to minimize treatment-related increases in body mass index, perhaps via increases in physical activity in the active treatment period, may prove useful in reducing fatigue in the posttreatment period.

\section{References}

Andrykowski, M. A., Curran, S. L., \& Lightner, R. (1998). Off-treatment fatigue in breast cancer survivors: A controlled comparison. Journal of Behavioral Medicine, 21, 1-18.

Bauer, D. J., \& Curran, P. J. (2003). Distributional assumptions of growth mixture models: Implications for overextraction of latent trajectory classes. Psychological Methods, 8, 338-363.

Berger, A. M. (1998). Patterns of fatigue and activity and rest during adjuvant breast cancer chemotherapy. Oncology Nursing Forum, 25, $51-62$.

Berger, A. M., \& Higginbotham, P. (2000). Correlates of fatigue during and following adjuvant breast cancer chemotherapy: A pilot study. Oncology Nursing Forum, 27, 1443-1448.
Berglund, G., Bolund, C., Fornander, T., Rutqvist, L. E., \& Sjoden, P. O. (1991). Late effects of adjuvant chemotherapy and postoperative radiotherapy on quality of life among breast cancer patients. European Journal of Cancer, 27, 1075-1081.

Bower, J. E., Ganz, P. A., Desmond, K. A., Bernaards, C., Rowland, J. H., Meyerowitz, B. E., \& Belin, T. R. (2006). Fatigue in long-term breast carcinoma survivors. Cancer, 106, 751-758.

Bower, J. E., Ganz, P. A., Desmond, K. A., Rowland, J. H., Meyerowitz, B. E., \& Belin, T. R. (2000). Fatigue in breast cancer survivors: Occurrence, correlates, and impact on quality of life. Journal of Clinical Oncology, 18, 743-753.

Broeckel, J. A., Jacobsen, P. B., Horton, J., Balducci, L., \& Lyman, G. H. (1998). Characteristics and correlates of fatigue after adjuvant chemotherapy for breast cancer. Journal of Clinical Oncology, 16, 1689-1696.

Butler, R. W., Damarin, F. L., Beaulieu, C., Schwebel, A. J., \& Thorn, B. E. (1989). Assessing cognitive coping strategies for acute postsurgical pain. Psychological Assessment, 1, 41-45.

Courneya, K. S., Mackey, J. R., Bell, G. J., Jones, L. W., Field, C. J., \& Fairey, A. S. (2003). Randomized controlled trial of exercise training in postmenopausal breast cancer survivors: Cardiopulmonary and quality of life outcomes. Journal of Clinical Oncology, 21, 1660-1668.

Cudeck, R., \& Henly, S. J. (2003). A realistic perspective on pattern representation in growth data: Comment on Bauer and Curran (2003). Psychological Methods, 8, 378-383.

de Jong, N., Candel, M. J. J. M., Schouten, H. C., Huijer Abu-Saad, H., \& Courtens, A. M. (2004). Prevalence and course of fatigue in breast cancer patients receiving adjuvant chemotherapy. Annals of Oncology, $15,896-905$.

Dimeo, F., Stieglitz, R. D., Novelli-Fischer, U., Fetscher, S., Mertelsmann, R., \& Keul, J. (1997). Correlations between physical performance and fatigue in cancer patients. Annals of Oncology, 8, 1251-1255.

Godin, G., Jobin, J., \& Bouillon, J. (1986). Assessment of leisure time exercise behavior by self-report: A concurrent validity study. Canadian Journal of Public Health, 77, 359-362.

Hann, D. M., Jacobsen, P. B., Azzarello, L. M., Martin, S. C., Curran, S. L., Fields, K. K., et al. (1998). Measurement of fatigue in cancer patients: Development and validation of the Fatigue Symptom Inventory. Quality of Life Research, 7, 301-310.

Helgeson, V. S., Snyder, P., \& Seltman, H. (2004). Psychological and physical adjustment to breast cancer over 4 years: Identifying distinct trajectories of change. Health Psychology, 23, 3-15.

Irwin, M. L., \& Ainsworth, B. E. (2004). Physical activity interventions following cancer diagnosis: Methodologic challenges to delivery and assessment. Cancer Investigation, 22, 30-50.

Jacobs, D. R., Jr., Ainsworth, B. E., Hartman, T. J., \& Leon, A. S. (1993). A simultaneous evaluation of 10 commonly used physical activity questionnaires. Medicine and Science in Sports and Exercise, 25, 81-91.

Jacobsen, P. B., Andrykowski, M. A., \& Thors, C. L. (2004). Relationship of catastrophizing to fatigue among women receiving treatment for breast cancer. Journal of Consulting and Clinical Psychology, 72, 355-361.

Jacobsen, P. B., Azzarello, L. M., \& Hann, D. M. (1999). Relationship of catastrophizing to fatigue severity in women with beast cancer. Cancer Research, Therapy, and Control, 8, 155-164.

Jacobsen, P. B., \& Stein, K. (1999). Is fatigue a long-term side effect of breast cancer treatment? Cancer Control, 6, 256-263.

Jensen, M. P., Turner, J. A., \& Romano, J. M. (2001). Changes in pain beliefs, catastrophizing, and coping are associated with improvement in multidisciplinary pain treatment. Journal of Consulting and Clinical Psychology, 69, 655-662.

Jones, B. L., Nagin, D. S., \& Roeder, K. (2001). A SAS procedure based on mixture models for estimating developmental trajectories. Sociological Methods and Research, 29, 374-393.

Kaplan, D., \& George, R. (1998). Evaluating latent variable growth models 
through ex post simulation. Journal of Educational and Behavioral Statistics, 23, 216-235.

Katz, J. N., Chang, L. C., Sangha, O., Fossel, A. H., \& Bates, D. W. (1996). Can comorbidity be measured by questionnaire rather than medical review? Medical Care, 34, 73-84.

Kumar, N., Allen, K. A., Riccardi, D., Bercu, B. B. Cantor, A., Minton, S., et al. (2004). Fatigue, weight gain, lethargy and amenorrhea in breast cancer patients on chemotherapy: Is subclinical hypothyroidism the culprit? Breast Cancer Research and Treatment, 83, 149-159.

Lankester, K. J., Phillips, J. E., \& Lawton, P. A. (2002). Weight gain during adjuvant and neoadjuvant chemotherapy for breast cancer: An audit of 100 women receiving FEC or CMF chemotherapy. Clinical Oncology, 14, 64-67.

Mast, M. E. (1998). Correlates of fatigue in survivors of breast cancer. Cancer Nursing, 21, 136-142.

McInnes, J. A., \& Knobf, M. T. (2001). Weight gain and quality of life in women treated with adjuvant chemotherapy for early-stage breast cancer. Oncology Nursing Forum, 28, 675-684.

Mock, V., Pickett, M., Ropka, M. E., Muscari, L. E., Stewart, K. J., Rhodes, V. A., et al. (2001). Fatigue and quality of life outcomes of exercise during cancer treatment. Cancer Practice, 9, 119-127.

Muthén, B. (2003). Statistical and substantive checking in growth mixture modeling: Comment on Bauer and Curran (2003). Psychological Methods, 8, 369-377.

Muthén, B. (2004). Latent variable analysis: Growth mixture modeling and related techniques for longitudinal data. In D. Kaplan (Ed.), The Sage handbook of quantitative methodology for the social sciences (pp. 345368). Thousand Oaks, CA: Sage.

Muthén, B., \& Muthén, L. (2004). Mplus User's Guide (v. 3.12). Los Angeles: Muthén \& Muthén.

National Comprehensive Cancer Network. (2003). Cancer-related fatigue clinical practice guidelines. Journal of National Comprehensive Cancer Network, 1, 308-332.

Nesselroade, J. R., \& Baltes, P. B. (1979). Longitudinal research in the study of behavior and development. New York: Academic Press.
Okuyama, T., Akechi, T., Kugaya, A., Okamura, H., Imoto, S., Nakano, T., et al. (2000). Supportive Care in Cancer, 8, 215-222.

Rindskopf, D. (2003). Mixture or homogenous? Comment on Bauer and Curran (2003). Psychological Methods, 8, 364-368.

Schwartz, A. L. (1998). Patterns of exercise and fatigue in physically active cancer survivors. Oncology Nursing Forum, 25, 485-491.

Servaes, P., Verhagen, C., \& Bleijenberg, G. (2002). Fatigue in cancer patients during and after treatment: Prevalence, correlates and interventions. European Journal of Cancer, 38, 27-43.

Servaes, P., Verhagen, C. A., \& Bleijenberg, G. (2002). Relations between fatigue, neuropsychological functioning, and physical activity after treatment for breast carcinoma. Cancer, 95, 2017-2026.

Servaes, P., Verhagen, S., \& Bleijenberg, G. (2002). Determinants of chronic fatigue in disease-free breast cancer patients: A cross-sectional study. Annals of Oncology, 13, 589-598.

Singer, J. D., \& Willett, J. B. (2003). Applied longitudinal data analysis: Modeling change and event occurrence. New York: Oxford University Press.

Sullivan, M. J. L., \& D'Eon, J. (1990). Relation between catastrophizing and depression in chronic pain patients. Journal of Abnormal Psychology, 99, 260-263.

Sullivan, M. J. L., Thorn, B., Haythornthwaite, J. A., Keefe, F., Martin, M., Bradley, L. A., et al. (2001). Theoretical perspectives on the relation between catastrophizing and pain. Clinical Journal of Pain, 17, $52-54$.

Theil, H. (1966). Applied economic forecasting. Amsterdam: NorthHolland.

Wessely, S., Hotopf, M., \& Sharpe, M. (1998). Chronic fatigue and its syndromes. New York: Oxford University Press.

Woo, B., Dibble, S. L., \& Piper, B. F. (1998). Differences in fatigue by treatment methods in women with cancer. Oncology Nursing Forum, 25, 915-920.

Wratten, C., Kilmurray, J., Nash, S., Seldon, M., Hamilton, C. S., O’Brien, P. C., et al. (2004). Fatigue during breast radiotherapy and its relationship to biological factors. International Journal of Radiation, Oncology, Biology, Physics, 59, 160-167. 